\title{
Is glucose/amino acid supplementation after exercise an aid to strength training?
}

\author{
A G Williams, $M$ van den Oord, A Sharma, D A Jones
}

\begin{abstract}
Background-The precise timing of carbohydrate and amino acid ingestion relative to a bout of resistance exercise may modulate the training effect of the resistance exercise.

Objective-To assess whether regular glucose/amino acid supplementation immediately after resistance exercise could enhance the gain in muscle strength brought about by resistance training. Methods-Seven untrained participants with a median age of 23 years and mean (SD) body mass 68.9 (13.5) $\mathrm{kg}$ resistance trained on a leg extension machine for five days a week for 10 weeks, using four sets of 10 repetitions. Alternate legs were trained on successive days, one leg each day. Subjects ingested either a supplement including $0.8 \mathrm{~g}$ glucose $/ \mathrm{kg}$ and $0.2 \mathrm{~g}$ amino acids/ $\mathrm{kg}$, or placebo, on alternate training days immediately after training. Therefore the supplement was always ingested after training the same leg (supplement leg). Isometric, isokinetic, and 1 repetition maximum (RM) strength were measured before, during, and after training. Blood samples were analysed to determine the acute responses of insulin and glucose to resistance exercise and supplementation or placebo.
\end{abstract}

Results-Serum insulin concentration peaked 20 minutes after supplement ingestion at ninefold the placebo level, and remained significantly elevated for at least 80 minutes $(p<0.01)$. Isometric, isokinetic, and 1 RM strength improved on both supplement and placebo legs $(p<0.05)$. There were no significant differences in the gain in strength between the supplement leg and the placebo leg $(p>0.05)$.

Conclusion-Regular glucose/amino acid supplementation immediately after resistance exercise is unlikely to enhance the gain in muscle strength brought about by resistance training.

(Br F Sports Med 2001;35:109-113)

Keywords: strength training; nutrition; amino acids; carbohydrate

Resistance training is widely used to improve sport performance, assist in rehabilitation from injury, enhance physical appearance, and improve health. Athletes such as body builders, who arguably perform more resistance training than any other athletes, typically use protein and amino acid supplements in an effort to improve the gains in muscle size and strength achieved by training. ${ }^{1}$ The efficacy of various supplementation practices is largely unknown.

Resistance exercise increases the rate of muscle protein turnover in humans by causing an acute increase in the rates of both muscle protein synthesis ${ }^{2-8}$ and muscle protein degradation. ${ }^{48}$ The balance between protein synthesis and degradation determines whether the net effect of a single exercise bout will be hypertrophy, no change, or atrophy of a muscle. Regular acute modification of net muscle protein synthesis after resistance exercise by influencing either synthesis or degradation, or both, will therefore impact on the rate and extent of muscle hypertrophy that can be achieved by chronic resistance training.

One way to increase net muscle protein synthesis after resistance exercise is by using nutritional supplements. Ingestion of either amino acids $^{910}$ or carbohydrates ${ }^{11}$ after resistance exercise has been shown to result in a more positive muscle protein balance over an acute period (a number of hours). The mechanism(s) of action are not clear, but could involve enhanced amino acid supply and thus enhanced intracellular availability of amino acids, ${ }^{9}{ }^{10}{ }^{12-14}$ the effects of raised concentrations of anabolic hormones such as insulin ${ }^{11} 15-$ ${ }_{17}$ or growth hormone, ${ }^{16}{ }^{17}$ or the effects of lowered concentrations of catabolic hormones such as cortisol $^{18}$ in limiting protein degradation for the purpose of gluconeogenesis. ${ }^{19}$

Just one recent abstract ${ }^{18}$ has reported the chronic effects of supplementation on muscle growth during resistance training. For 12 weeks, young men ingested either a $6 \%$ carbohydrate solution or a placebo during each exercise bout, and the carbohydrate supplemented group showed a significantly greater increase in muscle fibre cross sectional area than the placebo group. The findings suggest such a strong effect of supplementation that, according to calculations of statistical power, it should require no more than two subjects per group for the findings to be replicated with a significant effect.

The chronic effects of supplementation on the gain in muscle strength in response to resistance training have not yet been investigated. Therefore we studied the effect of regular supplementation immediately after exercise on the gain in muscle strength brought about by resistance training.

\section{Methods}

EXPERIMENTAL DESIGN

We used a within subject design to minimise intersubject variability in both training response and nutritional intake. Each subject in a 
"training" group served as his or her own control, in that one leg was randomly assigned to a "supplement leg" group, while the other leg was assigned to a "placebo leg" group. A second "non-training" group ingested the supplements during the 10 week intervention period but did no training of either leg.

SUBJECTS

Seven students (two women and five men) from the University of Birmingham volunteered to participate in the study, and formed the training group. Subject characteristics were median (range) age 23.0 (19-41) years and mean (SD) body mass and stature 68.9 (13.5) $\mathrm{kg}$ and $173.2(12.1) \mathrm{cm}$ respectively. Five further students (four women and one man) were recruited from the same population to form the non-training group; subject characteristics were median (range) age 22.0 (20-24) years and mean (SD) body mass and stature 63.8 (11.3) kg and $167.8(7.3) \mathrm{cm}$ respectively. All subjects were untrained, none having been involved in a resistance training programme for at least the previous six months. Each subject gave their full informed consent and all procedures were approved by the School of Sport and Exercise Sciences ethics subcommittee of the University of Birmingham.

\section{TRAINING}

The training group trained alternate legs on successive days - that is, one leg each day. The training involved unilateral leg extensions through a full range of motion on a Cybex VR2 training device five days a week (Monday to Friday) for 10 weeks, using four sets of 10 repetitions. Consequently, each leg received either two or three exercise bouts a week. The first set was performed using $75 \%$ of the 10 repetition maximum (RM) load, while the subsequent three sets were performed at $100 \%$ of the 10 RM load. Rest periods between sets were one minute. Loads were adjusted at the beginning of each week to maintain the $10 \mathrm{RM}$ training intensity.

\section{SUPPLEMENTATION}

Participants were blind to the following treatments: ingestion of either $500 \mathrm{ml}$ of an oral supplement containing $0.8 \mathrm{~g}$ glucose $/ \mathrm{kg}$ body mass and $0.2 \mathrm{~g}$ amino acids/kg (plus water, lemon flavouring, and colouring) or a placebo (designed to look and taste as much like the supplement as possible, containing $0.5 \mathrm{~g}$ dried milk powder, artificial sweetener, water, lemon flavouring, and colouring) on alternate training days immediately after training. Therefore the supplement was always ingested after training the same leg (supplement leg). The amino acids consisted of $17.1 \%$ glutamine, $11.0 \%$ leucine, $10.3 \%$ aspartic acid and $<10 \%$ each of another 15 amino acids. For a $70 \mathrm{~kg}$ person, the gross energy content of the supplement was about 1.2 MJ. The non-training group consumed the supplement, but not the placebo, on the equivalent days to the training group throughout the 10 weeks. A fast of at least two hours was required both before and after each training session (training group) or equivalent supplement ingestion (non-training group). For most participants, the most convenient method was to conduct the training and/or ingestion in the morning as a substitute for breakfast.

MEASUREMENTS

Three measures of strength of the quadriceps muscle group were used to monitor the effects of training and supplementation in the training group. Two of the three measures of strength were used to monitor the non-training group.

Isometric strength at $1.57 \mathrm{rad}$ of both knee and hip flexion ${ }^{20}$ was measured before and after the 10 week intervention period. A single test session involved at least three maximum voluntary contractions of each leg for up to five seconds each, with the highest force production of the three trials recorded as isometric strength at that test session. Between two and five test sessions separated by at least 48 hours were used before the intervention period to minimise the effects of learning on the observed changes over the 10 weeks, with the mean of the last two test sessions used to determine strength before the training began. The mean of two test sessions after the 10 weeks was used to determine strength after the training session. The repeatability of the measurements was good; the final two pretraining trials showed ratio limits of agreement ${ }^{21}$ of $1.00 \times / \div 1.09$. Also, percutaneous twitch superimposition ${ }^{22}$ during isometric contractions was used both before and after training to check that quadriceps activation levels were high and remained unchanged after training.

Isokinetic concentric strength of the quadriceps during knee extension at an angular velocity of $1.05 \mathrm{rad} / \mathrm{s}$ was measured using a Cybex 340 dynamometer before and after the 10 week intervention period. A single test session involved six maximal repetitions for each leg, with the highest torque production recorded as isokinetic strength at that test session. Between two and five test sessions separated by at least 48 hours were used before the intervention period to minimise the effects of learning on the observed changes over the 10 weeks, with the mean of the last two test sessions used to determine strength before training. The mean of two test sessions after the 10 weeks was used to determine strength after training. The repeatability of the measurements was good; the final two pretraining trials showed ratio limits of agreement of $0.98 \times / \div$ 1.13 .

The maximum load that could be lifted once (1 RM) on the Cybex VR2 training device such that the lower leg reached horizontal was determined for each leg at the beginning of each training week for the training group only.

Towards the end of the study, a cannula was inserted into a superficial forearm vein of five of the seven subjects (one woman, four men) from the training group, and blood samples were obtained immediately after and at 20 minute intervals until 120 minutes after the exercise bout on two separate days, once after supplementation and once after placebo. Blood was allowed to clot for 20 minutes, and serum 


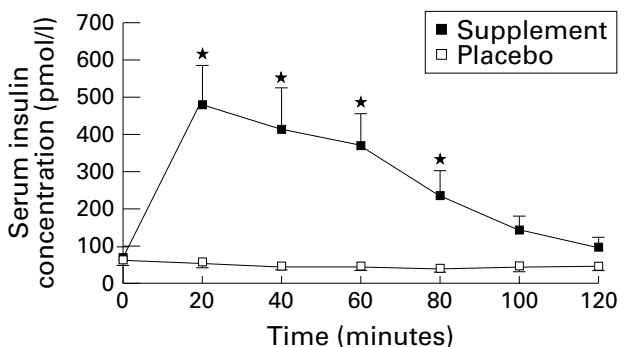

Figure 1 Acute effect of supplement and placebo on mean (SD) serum insulin concentration. ${ }^{\star}$ Significantly different from placebo result $(p<0.01)$.

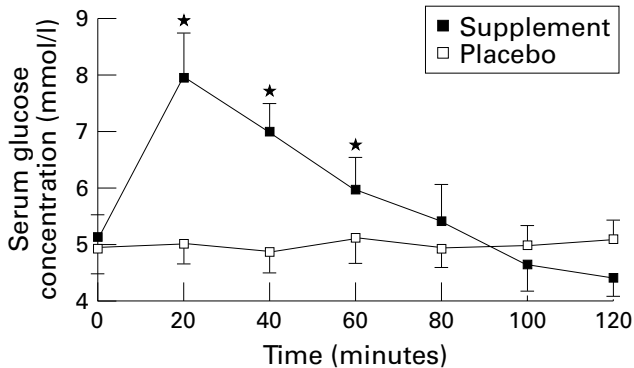

Figure 2 Acute effect of supplement and placebo on mean (SD) serum glucose concentration. * Significantly different from placebo result $(p<0.05)$.

was separated by centrifugation for storage at $-20^{\circ} \mathrm{C}$ until analysis within one month. Serum insulin concentrations were determined by double antibody ${ }^{125}$ I radioimmunoassay (Insulin RIA 100; Pharmacia \& Upjohn Diagnostics AB, Uppsala, Sweden), and serum glucose concentrations were determined by an enzymatic spectrophotometric method (Sigma Diagnostics, Poole, Dorset, UK). The coefficient of variation of the methods based on analysis of 70 duplicate samples were $11.2 \%$ and $7.0 \%$ for insulin and glucose respectively.

Body mass and percentage body fat ( $\%$ fat) estimated from skinfold measurements ${ }^{23}$ were determined both before and after training.

DATA ANALYSIS

Paired samples $t$ tests and analysis of variance with repeated measures were used to examine the effects of training on muscle strength and body morphology and the serum insulin responses to supplementation and placebo. Post hoc analysis was performed using Tukey's honestly significant difference test where appropriate. The accepted level of significance was $\mathrm{p}<0.05$.

Results

All results are presented as mean (SD). Serum insulin concentration peaked 20 minutes after ingestion of the supplement $(479$ (103) pmol/1) at over ninefold the placebo level (52 (11) $\mathrm{pmol} / \mathrm{l}$ ) and remained significantly higher than

Table 1 Mean (SD) strength of training group before and after training

\begin{tabular}{|c|c|c|c|c|c|c|}
\hline & \multicolumn{2}{|c|}{ Isometric $(N)$} & \multicolumn{2}{|c|}{ Isokinetic (N.m) } & \multicolumn{2}{|c|}{$1 \mathrm{RM}(\mathrm{kg})$} \\
\hline & Before & After & Before & After & Before & After \\
\hline Supplement leg & $574(75)$ & $633(84)^{\star}$ & $209(30)$ & $224(32)^{\star}$ & $40(6)$ & $56(8)^{\star}$ \\
\hline Placebo leg & $608(92)$ & $658(102)^{\star}$ & $213(31)$ & $226(35)^{\star}$ & $43(7)$ & $58(9)^{\star}$ \\
\hline
\end{tabular}

Significantly different from before training : ${ }^{\star} \mathrm{p}<0.05$.



Figure 3 Increase in mean (SD) strength in the supplement leg and the placebo leg during the training period.

after placebo ingestion for at least 80 minutes (fig 1). Insulin concentrations after ingestion of the supplement approached placebo values as the two hour monitoring period came to an end (95 (25) pmol/1 $v 38$ (7) pmol/1). Serum glucose concentration also peaked 20 minutes after ingestion of the supplement (7.9 (0.8) mmol/1) compared with an unchanged concentration in the placebo condition (5.0 (0.4) $\mathrm{mmol} / \mathrm{l}$ ) and remained significantly higher than after placebo ingestion for at least 60 minutes (fig 2).

The training group improved during training in each of the isometric, isokinetic, and $1 \mathrm{RM}$ strength tests on both supplement and placebo legs (table 1). The non-training group did not change in isometric strength (449 (72) $v 450$ (69) $\mathrm{N}$ ), but showed a significant $5.5 \%$ reduction in isokinetic strength (163 (24) $v 154$ (22) N.m). Muscle activation levels during isometric contractions $(92-100 \%)$ showed only minimal changes from before to after the intervention period and did not differ between supplement and placebo legs, indicating that all participants could achieve near maximal activation of their quadriceps muscle groups.

For the training group, there were no significant differences in the improvements in isometric strength $(p=0.46)$, isokinetic strength $(\mathrm{p}=0.28)$, or $1 \mathrm{RM}$ strength $(\mathrm{p}=$ 0.25 ) between the supplement leg and the placebo leg. However, there did appear to be some trend towards a benefit of supplementation in that there were about 33\% greater improvements in the supplement leg compared with the placebo leg on each measure of strength (fig 3). The rate of change in $1 \mathrm{RM}$ strength of the two legs over the 10 week period (fig 4) also

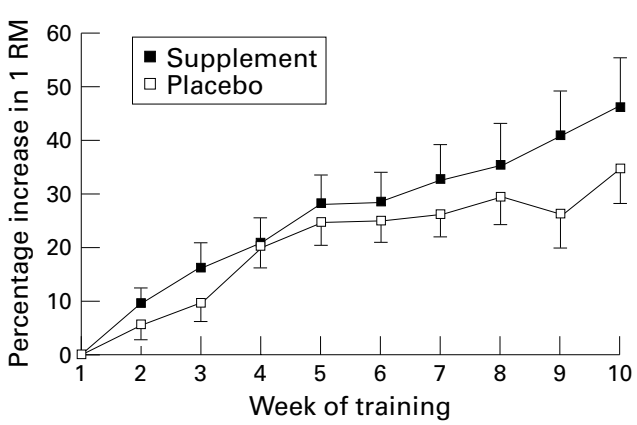

Figure 4 Effect of training on mean (SD) 1 RM strength in the supplement leg and the placebo leg. 
illustrates the possibility of a trend towards a benefit of supplementation.

Body mass did not change from before to after the intervention period for either the training group (68.9 (5.1) $\mathrm{kg} v 68.8$ (4.7) $\mathrm{kg}, \mathrm{p}=0.86$ ) or the non-training group (63.8 (5.0) $\mathrm{kg} v 63.8$ (5.2) $\mathrm{kg}, \mathrm{p}=0.96$ ). Similarly, percentage body fat did not change from before to after the intervention period for either the training group (16.0 (3.5)\% fat $v 16.4(3.4) \%$ fat, $\mathrm{p}=0.51$ ) or the non-training group (19.5 (2.5)\% fat $v 20.8$ $(2.7) \%$ fat, $\mathrm{p}=0.14)$. Concurrently, there were no differences between the training and nontraining groups in the response to the intervention period for either body mass $(p=0.88)$ or percentage body fat $(p=0.29)$.

\section{Discussion}

The strength increases in the present study were comparable with, or somewhat lower than, those reported by others. ${ }^{24-29}$ It is likely that the strength gains reported here were relatively modest because of the low training volume in this study (due partly to the alternate day training regime) and variations in testing protocols between studies. Large differences between increases in $1 \mathrm{RM}$ and other measures of strength as in this study have been reported previously, ${ }^{24} 250$ and probably reflect the differing roles of neural adaptation within the central nervous system and changes in the intrinsic strength of the muscle groups. ${ }^{24}$ The nontraining group did not improve muscle strength, which confirmed that simply ingesting the supplements while doing no training had no beneficial effect.

Supplementation after exercise significantly elevated serum insulin and glucose levels above baseline and above placebo levels, as expected. Previous studies have shown elevations of insulin and glucose of broadly similar magnitude during or after resistance exercise and supplementation. ${ }^{11}{ }^{15-17}$

It is not surprising that the kinanthropometric measures used in this study did not show any differences within or between the training and non-training groups in response to the intervention period. The training volume was relatively low, and evidently was not sufficient to produce changes in whole body variables such as body fat.

The main finding of this study is that regular supplementation after exercise with a combination of glucose and amino acids did not significantly enhance the gain in muscle strength brought about by resistance training. This finding is in contrast with that of Tarpenning et al, ${ }^{18}$ who reported a significant benefit of carbohydrate supplementation on muscle growth. The data presented here do not support the use of regular after exercise supplementation to increase the training induced gains in muscle strength. However, there were trends towards a greater gain in strength by the supplemented leg, and for an increasing benefit of supplementation as the duration of training increased-when muscle hypertrophy would have become a gradually more important factor in increasing strength. ${ }^{24}$ Therefore it may be premature to completely dismiss the use of supplementation after exercise to increase training induced gains in muscle strength.

The within subject design of this study was used to minimise intersubject differences in both training response and nutritional intake. However, this approach assumes an independent effect (or at least a greater effect) of the supplementation on the supplement leg. The premise of this approach is that there is a critical period immediately after resistance exercise when supplementation has greatest potential to influence protein metabolism. ${ }^{14}$ However, this has not yet been shown conclusively, ${ }^{31}$ and further research into protein kinetics after resistance exercise both with and without supplementation is necessary.

\section{CONCLUSIONS}

Regular supplementation that raises insulin immediately after resistance exercise does not enhance gains in muscle strength significantly over 10 weeks of resistance training. This suggests that athletes and others who wish to gain muscle strength and mass by resistance training would not benefit if they accompany each training session with supplementation. However, some trends in the data suggest that it may be premature to completely dismiss the concept of supplementation after exercise to increase training induced gains in muscle strength.

A W is grateful to Optimal Performance Limited for research funding which supported this study.

1 Brill JB, Keane MW. Supplementation patterns of competitive male and female bodybuilders. Int $\mathcal{f}$ Sport Nutr 1994;4:398-412.

2 Chesley A, MacDougall JD, Tarnopolsky MA, et al. Changes in human muscle protein synthesis after resistance exercise. f Appl Physiol 1992;73:1383-8.

3 Yarasheski KE, Zachwieja JJ, Bier, DM. Acute effects of resistance exercise on muscle protein synthesis rate in young and elderly men and women. Am f Physiol 1993;273:E210-14

4 Biolo G, Maggi SP, Williams BD, et al. Increased rates of muscle protein turnover and amino acid transport after
resistance exercise in humans. Am $\mathcal{f}$ Physiol 1995;268: resistance

5 MacDougall JD, Gibala MJ, Tarnopolsky MA, et al. The time course for elevated muscle protein synthesis following heavy resistance exercise. Can F Appl Physiol 1995;20:4806.

6 Tipton KD, Ferrando AA, Williams BD, et al. Muscle protein metabolism in female swimmers after a combination of resistance and endurance exercise. F Appl Physiol 1996;81: 2034-8

7 Phillips SM, Tipton KD, Aarsland A, et al. Mixed muscle protein synthesis and breakdown after resistance exercise in humans. Am f Physiol 1997;273:E99-107.

8 Phillips SM, Tipton KD, Ferrando AA, et al. Gender does not affect muscle protein turnover following resistance not affect muscle protein turnover following

9 Tipton KD, Ferrando AA, Phillips SM, et al. Response of muscle protein metabolism to ingestion of an oral amino muscle protein metabolism to ingestion of an oral amino 1996;39:A12.

10 Tipton KD, Ferrando AA, Phillips SM, et al. Muscle protein metabolism during ingestion of essential amino acids afte resistance exercise. Med Sci Sports Exerc 1997;29:S295.

11 Roy BD, Tarnopolsky MA, MacDougall JD, et al. Effect of glucose supplement timing on protein metabolism after resistance training. F Appl Physiol 1997;82:1882-8.

12 MacLean DA, Kiens B, Rohde T, et al. Branched chain amino acid supplementation reduces muscle amino acid release after eccentric exercise. Med Sci Sports Exerc 1996; 28:S181.

13 Tipton KD, Wolfe RR. Exercise-induced changes in protein metabolism. Acta Physiol Scand 1998;162:377-87.

14 Biolo G, Tipton KD, Klein S, et al. An abundant supply of amino acids enhances the metabolic effect of exercise on muscle protein. Am f Physiol 1997;273:E122-9.

15 Fahey TD, Hoffman K, Colvin W, et al. The effects of intermittent liquid meal feeding on selected hormones and substrates during intense weight training. Int $\mathcal{f}$ Sport Nutr 1993;3:67-75. 
16 Chandler RM, Byrne HK, Patterson JG, et al. Dietary supplements affect the anabolic hormones after weighttraining exercise. $\mathcal{F} A P P$

17 Kraemer WJ, Volek JS, Bush JA, et al. Hormonal responses to consecutive days of heavy-resistance exercise with or without nutritional supplementation. F Appl Physiol 1998; 73:1383-8

18 Tarpenning KM, Wiswell RA, Marcell TJ, et al. Influence of weight training exercise and modification of hormonal response on skeletal muscle growth. Med Sci Sports Exerc 1998;30:S227.

19 Simmons PS, Miles JM, Gerich JE, et al. Increased proteolysis: an effect of increases in plasma cortisol within the physiological range. $\mathcal{F}$ Clin Invest 1984;73:412-20.

20 Edwards RHT, Young A, Hosking GP, et al. Human skeletal muscle function: description of tests and normal values. Clin Sci Mol Med 1977;52:283-90.

21 Nevill AM, Atkinson G. Assessing agreement between measurements recorded on a ratio scale in sports medicine measurements recorded on a ratio scale in sports medir

22 Rutherford OM, Jones DA, Newham DJ. Clinical and experimental application of the percutaneous twitch superexperimental application of the percutaneous twitch superimposition technique for the study of human muscle
activation. 7 Neurol Neurosurg Psychiatry 1986;49:1288-91.

23 Durnin JVGA, Womersley J. Body fat assessed from total body density and its estimation from skinfold thickness: measurements on 481 men and women aged from 16 to 72 years. Br F Nutr 1974;32:77-97.
24 Rutherford OM, Jones DA. The role of learning and coordination in strength training. Eur F Appl Physiol 1986;55: $100-5$.

25 Rutherford OM, Greig CA, Sargeant AJ, et al. Strength training and power output: transference effects in the human quadriceps muscle. F Sports Sci 1986;4:101-7.

26 Braith RW, Graves JE, Pollock ML, et al. Comparison of 2 vs 3 days/week of variable resistance training during 10- and 18-week programs. Int $\mathcal{F}$ Sports Med 1989;10:450-4.

27 Sale DG, Martin JE, Moroz DE. Hypertrophy without increased isometric strength after weight training. Eur $\mathcal{F}$ Appl Physiol 1992;64:51-5.

28 Hisaeda H, Miyagawa K, Kuno S, et al. Influence of two different modes of resistance training in female subjects. Ergonomics 1996;39:842-52.

29 Starkey DB, Pollock ML, Ishida Y, et al. Effect of resistance training volume on strength and muscle thickness. Med Sci Sports Exerc 1996;28:1311-20.

30 Baker D, Wilson G, Carlyon B. Generality versus specificity: a comparison of dynamic and isometric measures of strength and speed-strength. Eur f Appl Physiol 1994;68: 350-5.

31 Rasmussen BB, Tipton KD, Miller SL, et al. An oral essential amino acid-carbohydrate supplement enhances muscle protein anabolism after resistance exercise. $\mathcal{F}$ Appl Physiol 2000;88:386-92.

\section{Take home message}

Ingesting a carbohydrate/protein supplement immediately after strength training is unlikely to enhance the gains in muscle strength. However, supplementation would have no obvious detrimental effect, would speed the restoration of muscle glycogen stores, and may possibly increase the training effects in some situations.

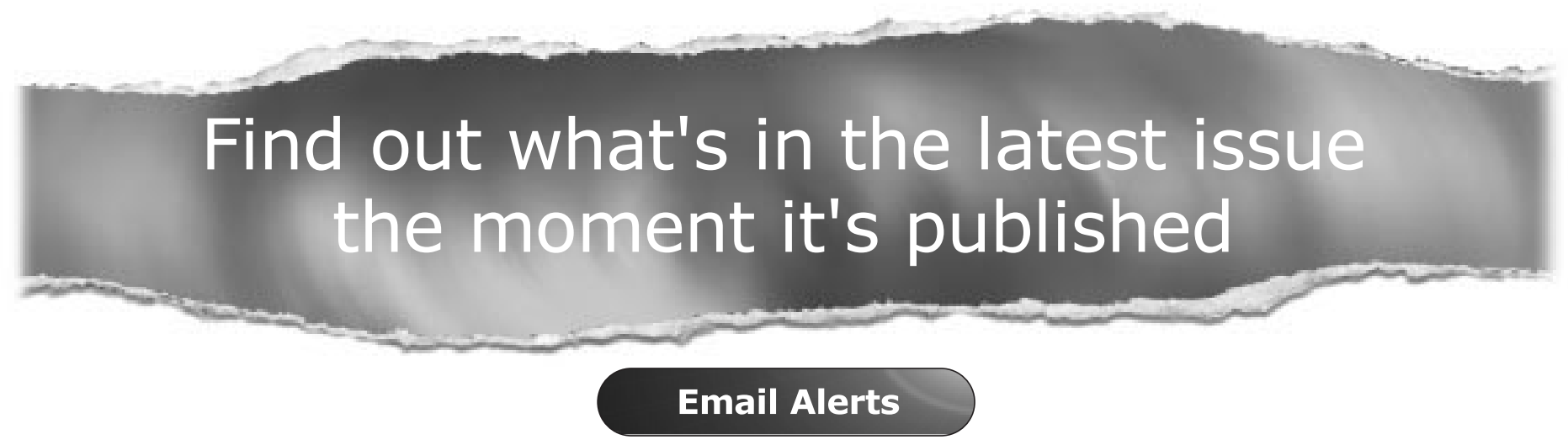

Sign up to receive the table of contents by email every month. You can select from three alerts:

Table of Contents (full), TOC Awareness (notice only); British Journal of Sports Medicine related announcements.

\section{www.bjsportmed.com}

